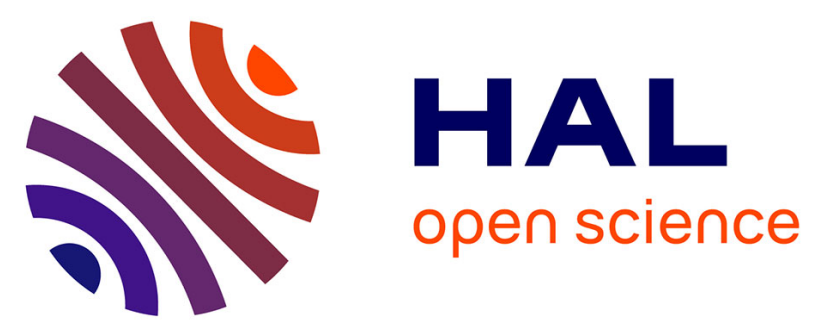

\title{
Statistical learning multiobjective optimization for large-scale achromatic metalens at visible regime
}

Mahmoud M R Elsawy, Mickael Binois, Régis Duvigneau, Stéphane Lanteri, Patrice Genevet

\section{- To cite this version:}

Mahmoud M R Elsawy, Mickael Binois, Régis Duvigneau, Stéphane Lanteri, Patrice Genevet. Statistical learning multiobjective optimization for large-scale achromatic metalens at visible regime. CLEO, Laser Science to Photonic Applications, May 2021, San Jose, California (web conference format), United States. 10.1364/CLEO_QELS.2021.FTh2M.3 . hal-03357023

\section{HAL Id: hal-03357023 \\ https://hal-unilim.archives-ouvertes.fr/hal-03357023}

Submitted on 28 Sep 2021

HAL is a multi-disciplinary open access archive for the deposit and dissemination of scientific research documents, whether they are published or not. The documents may come from teaching and research institutions in France or abroad, or from public or private research centers.
L'archive ouverte pluridisciplinaire HAL, est destinée au dépôt et à la diffusion de documents scientifiques de niveau recherche, publiés ou non, émanant des établissements d'enseignement et de recherche français ou étrangers, des laboratoires publics ou privés. 


\title{
Statistical learning multiobjective optimization for large-scale achromatic metalens at visible regime
}

\author{
Mahmoud M. R. Elsawy, Mickaël Binois, Régis Duvigneau, Stéphane Lanteri, and Patrice Genevet \\ Université Côte d'Azur, Inria, CNRS, LJAD, 06902 Sophia Antipolis Cedex, France \\ CNRS, CRHEA, Université Côte d'Azur, Sophia Antipolis, Valbonne, France \\ mahmoud.elsawy@inria.fr
}

\begin{abstract}
A novel computational methodology based on statistical learning multiobjective optimization is developed to optimize large-scale achromatic 3D metalenses in the visible regime. The optimized lens has a numerical aperture of 0.56 and an average focusing efficiency of $45 \%$.
\end{abstract}

\begin{abstract}
The metasurface is a nanostructured interface with a subwavelength thickness which manipulates light through spatially arranged meta-atoms. These meta-atoms ensure adequate control of the light attributes by altering the phase, amplitude and polarization of the incoming wavefront [1-2]. Due to the versatility of the metasurface concept, peculiar and outstanding applications have been demonstrated recently [3-4]. Yet, the precise engineering of the subwavelength meta-atoms, in particular for extremely challenging applications, is therefore crucial for improving the performance. As a result, numerous inverse design methodologies have recently been adopted to further broaden the potential of a metasurface design, in particular for single-objective based devices [5].
\end{abstract}

Nevertheless, the next decade is expected to witness a notable increase in the field of multifunctional metasurfaces owing to the increasing demand in the design of flat optical devices with a diverse variety of functionalities. This makes it a mandatory step for the next metasurface generation to leverage robust inverse design techniques, which are capable of optimizing metasurfaces with multiple functionalities. However, this is not an easily manageable task, since the optimization of multifunctional metasurfaces requires a solution to an optimization problem with different conflicting objectives and potentially entails a large parameter space.

Fig.1: (a) schematic view of the 3D achromatic metalens in the $\mathrm{x}-\mathrm{z}$ plane. The metalens aims at focusing the three colours at the same focal distance F. The diameter of the metasurface is $\mathrm{D}$ and the thickness is fixed as $\mathrm{h}=1000$ $\mathrm{nm}$. (b) one-quarter of the geometry at the $x-y$ plane. The 16 optimization parameters are defined as follows; 10 diameters represented by the yellow cylinders and 6 parameters for the distances between the outer rings (black arrows). (c) refers to the intensity profile cut along the propagation direction $\mathrm{z}$ for one of the optimized designs with NA=0.47.
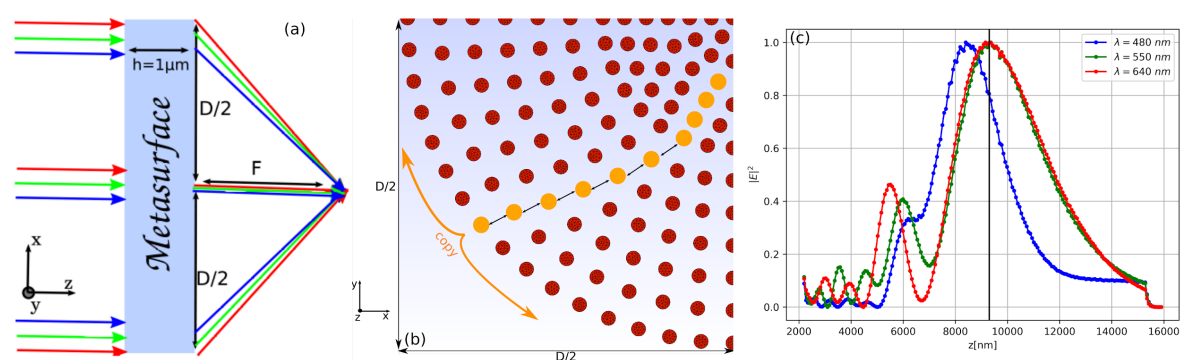

In these circumstances, the development of multiobjective evolutionary algorithms is a widespread methodology. The numerical methods relying on this procedure converge to the global set of solutions and can also accommodate broad parameters owing to their stochastic nature. They are, however, computationally demanding and subjected require a high number of evaluations of the objective functions [6]. This is an impractical limit when combined with an expensive 3D electromagnetic solver required for simulating realistic devices.

In this work, we present for the first time to the metasurface community a multiobjective optimization approach based on statistical learning. This method is based on surrogate modelling, which replaces the high fidelity electromagnetic evaluation process with a simpler and cheaper model for the prediction of the new designs during the optimization process. Even though it converges to the global set of solutions, it requires fewer iterations compared to the classical global evolutionary strategies [7]. We combine this approach with our in-house developed 3D electromagnetic solver based on the Discontinuous Galerkin Time-Domain (DGTD) method [8] to optimize 3D achromatic metalens with numerical aperture NA $>0.5$. Our metalens attempts to focus the RGB colours at the same focal plane with the maximum feasible focusing efficiency (see Fig. 1(a)). In other words, we seek to get the most suitable compromise between the chromatic dispersion and the focusing efficiency for a given fixed focal distance. 
Our 3D lens is composed of concentric rings of cylindrical nanopillars represented by the red colour in Fig. 1(b). The height of the cylinders is fixed as $1000 \mathrm{~nm}$. As a first design, we consider 12 concentric rings (apart from the central cylinder), where the number of the cylinders in each ring (fixed during the optimization) increases towards the outer rings. In order to reduce the computational cost, we assume that the cylinders in each ring share the same diameter. Consequently, we consider optimizing one row of the cylinders along the radial direction together with the distances between the rings. The 16 optimization parameters are indicated in Fig.1 (b) by the yellow cylinders and the black arrows, for the diameters and the distances, respectively. In Fig. 1(c), we present the intensity profile along the propagation direction $\mathrm{z}$ for one of the optimized designs, where the NA=0.47. As can be seen, the three colours are focused nearly at the predefined focal plane (represented by the vertical black line). The average focal error for the three wavelengths is less than $13 \%$. The average efficiency of our 3D metalens with $\mathrm{NA}=0.47$ is roughly $56 \%$.

Fig 2: (a) schematic view of the extended 3D achromatic metalens. Four additional rings are added to the optimized design shown in Fig. 1(b). The numerical aperture of the new optimized design is $\mathrm{NA}=0.56$. The average focusing efficiency for the three wavelengths is approximately $45 \%$. (b) gives the cut of the intensity profile along the propagation direction. The vertical black line refers to the position
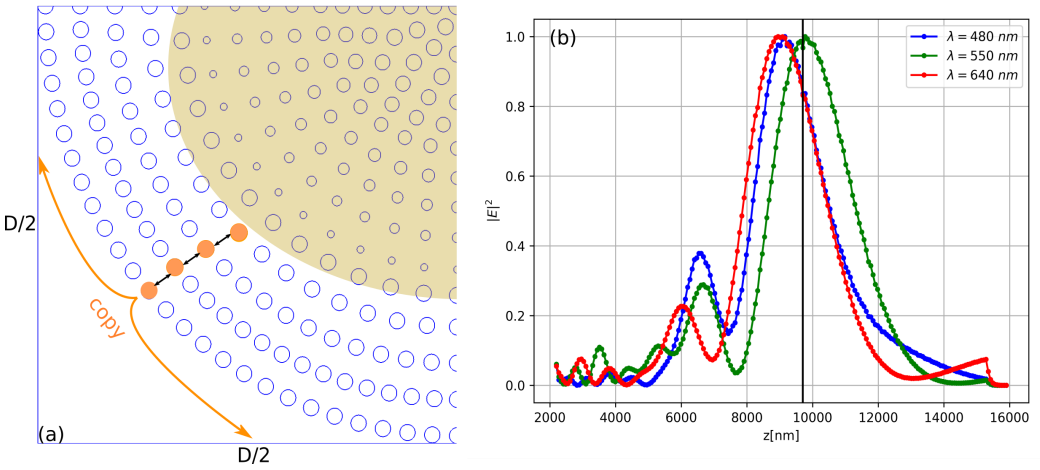
of the focal plane along $\mathrm{z}$.

We further increased the numerical aperture by adding four additional rings (Fig. 2(a)) to the metalens while keeping the central 11 rings from the former design depicted in Fig. 1 (b). The proposed extra rings enlarge the diameter of the lens, hence renders more dispersion control for the three wavelengths. We found that the relative focal error for the three wavelengths is reduced to $5 \%$, which indicates that the chromatic dispersion is diminished as depicted in Fig. 2(b). Interestingly, the average focusing efficiency for this $3 \mathrm{D}$ lens with NA=0.56 is $45 \%$. To the best of our knowledge, this is the highest focusing efficiency obtained so far for such as 3D metalens architecture in the visible regime.

\section{References}

[1] Yu, N.; Genevet, P.; Kats, M. A.; Aieta, F.; Tetienne, J.-P.; Capasso, F.; Gaburro, Z. "Light Propagation with Phase Discontinuities: Generalized Laws of Reflection and Refraction”. Science 2011, 334, 333-337.

[2] Lin, D.; Fan, P.; Hasman, E.; Brongersma, M. L. "Dielectric gradient metasurface optical elements". Science 2014, 345, 298-302.

[3] Krasnok, A.; Tymchenko, M.; Alù, A. "Nonlinear metasurfaces: a paradigm shift in nonlinear optics”. Materials Today 2018, 21,8 -21.

[4] Bekenstein, R.; Pikovski, I.; Pichler, H.; Shahmoon, E.; Yelin, S.; Lukin, M. "Quantum metasurfaces with atom arrays”. Nature Physics 2020, 16, 676-681.

[5] Elsawy, M. M. R.; Lanteri, S.; Duvigneau, R.; Fan, J. A.; Genevet, P. "Numerical Optimization Methods for Metasurfaces”. Laser \& Photonics Reviews 2020, 14, 1900445.

[6] Whiting, E. B.; Campbell, S. D.; Kang, L.; Werner, D. H. Meta-atom library generation via an efficient multi-objective shape optimization method. Opt. Express 2020, 28, 24229-24242.

[7] Elsawy, M. M. R.; Lanteri, S.; Duvigneau, R.; Brière, G.; Mohamed, M. S.; Genevet, P. Global optimization of metasurface designs using statistical learning methods. Scientific Reports 2019, 9.

[8] DIOGENES: A Discontinuous-Galerkin based software suite for nano-optics. https://diogenes.inria.fr/. 